\title{
Optimal Variational Method for Truly Nonlinear Oscillators
}

\author{
Vasile Marinca $^{1,2}$ and Nicolae Herişanu ${ }^{1,2}$ \\ ${ }^{1}$ Faculty of Mechanical Engineering, "Politehnica” University of Timişoara, Bd. M. Viteazu, 1, 300222 Timişoara, Romania \\ ${ }^{2}$ Center of Advanced Research in Engineering Sciences, Romanian Academy, Timişoara Branch, \\ Bd. M. Viteazu, 24, 300223, Timişoara, Romania
}

Correspondence should be addressed to Vasile Marinca; vmarinca@mec.upt.ro

Received 6 August 2012; Accepted 19 November 2012

Academic Editor: Kale Oyedeji

Copyright (c) 2013 V. Marinca and N. Herişanu. This is an open access article distributed under the Creative Commons Attribution License, which permits unrestricted use, distribution, and reproduction in any medium, provided the original work is properly cited.

The Optimal Variational Method (OVM) is introduced and applied for calculating approximate periodic solutions of "truly nonlinear oscillators". The main advantage of this procedure consists in that it provides a convenient way to control the convergence of approximate solutions in a very rigorous way and allows adjustment of convergence regions where necessary. This approach does not depend upon any small or large parameters. A very good agreement was found between approximate and numerical solution, which proves that OVM is very efficient and accurate.

\section{Introduction}

It is known that the study of nonlinear differential equations is restricted to a variety of special classes of equations and the method of solution usually involves one or more techniques to achieve analytical approximations to the solutions. Many researchers have recently paid much attention to find and develop approximate solutions. Perturbation methods have been successfully employed to determine approximate solutions to weakly nonlinear problems [1-3]. But the use of perturbation theory in many problems is invalid or it simply breaks down for parameters beyond a certain specified range. Therefore, new analytical techniques should be developed to overcome these shortcomings. Such a new technique should work over a large range of parameters and yield accurate analytical approximate solutions beyond the coverage and ability of the classical perturbation methods. There has been a great need for effective algorithms to avoid the work required by traditional techniques, but it is difficult to obtain convergent results in the cases of strong nonlinearity.

Recently, many new approaches have been proposed for this purpose, such as various modified Lindstedt-Poincare methods [4], some linearization methods [5, 6], the Adomian decomposition method [7], the optimal homotopy asymptotic method $[8,9]$, the optimal variational iteration method
[10], the energy balance method [11], and so on. A variational principle for nonlinear oscillations by constructing the Hamiltonian was studied in [11]. Variational principles have a great importance in physics and engineering since they establish connections between these disciplines and their applications are useful in devising various approximate techniques. Variational methods have been and continue to be popular tools for nonlinear analysis. They combine physical insights into the nature of the solution of the problem and the solutions obtained using possible trial functions are the best. It is known that computing a Lagrangian for dynamical systems with more general Newtonian forces is nowadays applicable only to systems with force derivable from a potential function (basically, conservative systems). Strictly speaking, conservative dynamical systems do not exist in our Newtonian environment. As a result, the Lagrangian representation of conservative Newtonian systems is in general only a crude approximation of physical reality. The problem of the existence of a Lagrangian, Hamiltonian, or Routhian can be studied today with a variety of modern and sophisticated mathematical tools which include the use of functional analysis, prolongation theory, and differential geometry, to cite only a few. This question is called "Inverse Problem in Newtonian Mechanics" [12] and consists in the identification of the methods for the construction of a 
Lagrangian, Hamiltonian, or Routhian form given equations of motion.

In this paper we construct accurate approximations to periodic solutions and frequencies of the so-called truly nonlinear oscillator (TNO). Following Mickens and Oyedeji $[13,14]$, the most general form of a TNO is given by the following differential equation:

$$
\ddot{x}+g(x)=h f(x, \dot{x}), \quad x(0)=A, \dot{x}(0)=0,
$$

where the dot denotes the derivative with respect to variable $t, A$ is a positive arbitrary parameter, and the functions $g(x)$ and $f(x, \dot{x})$ have the properties:

$$
\begin{gathered}
g(-x)=-g(x), \\
f(-x,-\dot{x})=-f(x, \dot{x}),
\end{gathered}
$$

and $g(x)$ does not have for small $x$ a dominant term proportional to $x$.

In the present work, we consider $g(x)=a x^{1 / 3}+b x^{3}$, $f(x, \dot{x})=0$ and therefore the truly nonlinear oscillator is modeled by the following nonlinear differential equation:

$$
\ddot{x}+a x^{1 / 3}+b x^{3}=0
$$

subject to initial conditions

$$
x(0)=A, \quad \dot{x}(0)=0,
$$

where $a, b$, and $A$ are known parameters.

In (3), there exists no small or large parameter.

\section{Basic Idea of Optimal Variational Method and Solution}

In order to develop an application of the OVM, we consider the following differential equation:

$$
\ddot{x}+g(x)=0
$$

with the initial conditions given by (4) and gbeing an arbitrary odd function.

Introducing a new independent variable $\tau$ and a new unknown $x(t)$ as

$$
\tau=\Omega t, \quad x(t)=A u(\tau),
$$

where $\Omega$ is the frequency of the system (5), then this becomes

$$
\Omega^{2} u^{\prime \prime}+A^{-1} g(A u)=0,
$$

where prime denotes derivative with respect to the new variable $\tau$.

The initial conditions (4) become

$$
u(0)=1, \quad u^{\prime}(0)=0 .
$$

The variational principle for (7) can be easily established if there exists a function

$$
J=\int_{0}^{\pi / 2} L\left(\tau, u, u^{\prime}\right) d \tau
$$

which admits as extremals the solutions of (7) and (8) where $L$ is the Lagrangian of the system (7):

$$
L\left(\tau, u, u^{\prime}\right)=-\frac{1}{2} \Omega^{2} u^{\prime 2}+A^{-1} G(u) .
$$

The function $G(u)$ is given by the equation

$$
\frac{d G(u)}{d u}=g(A u) \text {. }
$$

We assume that the approximate periodic solutions of (7) and (8) can be expressed as

$$
\bar{u}(\tau)=\sum_{k=1}^{m} C_{k} \cos (2 k-1) \tau,
$$

where $C_{k}$ are arbitrary unknowns at this moment and $m$ is a positive integer number. Choosing the solution (12) has been made in accordance to the properties (2).

Substituting (12) into (9) results in

$$
J\left(C_{1}, C_{2}, \ldots, C_{m}, \Omega\right)=\int_{0}^{\pi / 2}\left[-\frac{1}{2} \Omega^{2} u^{\prime 2}+A^{-1} G(u)\right] d \tau .
$$

Applying the Ritz method [9], we require

$$
\frac{\partial J}{\partial C_{1}}=\frac{\partial J}{\partial C_{2}}=\cdots=\frac{\partial J}{\partial C_{m}}=0 .
$$

From (14) and from the initial condition $\left(8_{1}\right)$ which becomes

$$
C_{1}+C_{2}+\cdots+C_{m}=1
$$

we can obtain optimally the parameters $C_{i}, i=1,2, \ldots, m$, and the frequency $\Omega$.

We remark that the choice of the approximate solution (12) is not unique. We can alternatively choose another expression of the approximate periodic solution in the form

$$
\bar{u}(\tau)=\sum_{k=1}^{m} C_{k}^{*} \cos (4 k-1) \tau
$$

and so on. With the parameters $C_{i}$ (called convergencecontrol parameters) and the frequency $\Omega$ known, the approximate periodic solutions is well determined.

The validity of the proposed approach is illustrated on the TNO given by (3). Using the transformations (6), equation (3) can be written in the form

$$
\Omega^{2} u^{\prime \prime}+a A^{-2 / 3} u^{1 / 3}+b A^{2} u^{3}=0
$$

and the Lagrangian becomes

$$
L\left(\tau, u, u^{\prime}\right)=-\frac{1}{2} \Omega^{2} u^{\prime 2}+\frac{3}{4} a A^{-2 / 3} u^{4 / 3}+\frac{1}{4} b A^{2} u^{4} .
$$

If we consider $m=3$ into (12), then the approximate periodic solutions become

$$
\bar{u}(\tau)=C_{1} \cos \tau+C_{2} \cos 3 \tau+C_{3} \cos 5 \tau .
$$




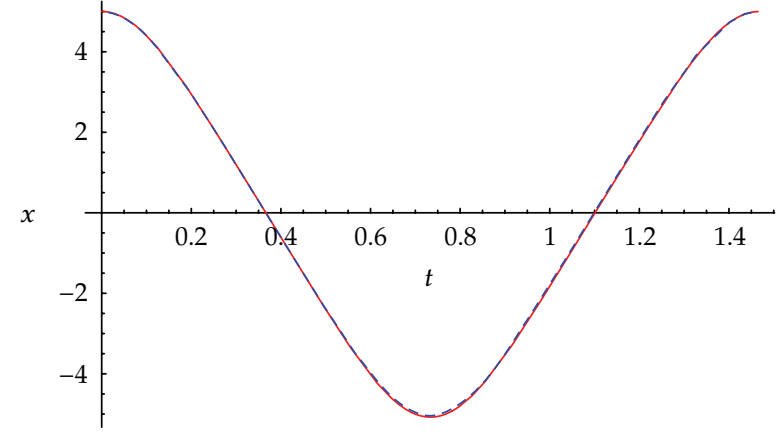

FIgURE 1: Comparison between the approximate solution (27) and numerical solution of (3) in the case $a=b=1, A=5$, red solid line: numerical integration results, blue dashed line: approximate solution.

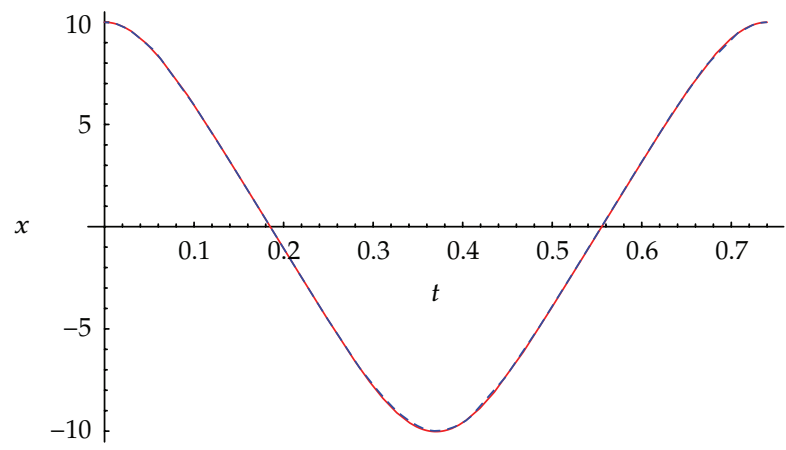

FIGURE 2: Comparison between the approximate solution (29) and numerical solution of (3) in the case $a=b=1, A=10$, red solid line: numerical integration results, blue dashed line: approximate solution.

Now, substituting (19) into (18) and (13), we have successively

$$
\begin{aligned}
\int_{0}^{\pi / 2} \bar{u}^{\prime 2} d \tau=\frac{\pi}{4}( & \left.C_{1}^{2}+9 C_{2}^{2}+25 C_{3}^{2}\right) \\
\int_{0}^{\pi / 2} \bar{u}^{4} d \tau=\frac{\pi}{16}[ & 3\left(C_{1}^{4}+C_{2}^{4}+C_{3}^{4}\right) \\
+ & 12\left(C_{1}^{2} C_{2}^{2}+C_{1}^{2} C_{3}^{2}+C_{2}^{2} C_{3}^{2}\right) \\
+ & \left.4 C_{1}^{3} C_{2}+12 C_{1}^{2} C_{2} C_{3}+12 C_{1} C_{2}^{2} C_{3}\right]
\end{aligned}
$$

To calculate the middle term $u^{4 / 3}$ into (18) we use the series expansion [15]:

$$
\begin{gathered}
\cos ^{1 / 3} \tau=\alpha\left(\cos \tau-\frac{1}{5} \cos 3 \tau+\frac{1}{10} \cos 5 \tau\right. \\
-\frac{7}{110} \cos 7 \tau+\frac{1}{22} \cos 9 \tau \\
\left.-\frac{13}{374} \cos 11 \tau+\cdots\right),
\end{gathered}
$$

where $\alpha=1.15959526696393$. If we denote $H(\bar{u})=\bar{u}^{4 / 3}$ where $\bar{u}$ is given by (19), then using the relation

$$
H\left(\bar{u}_{0}+h\right)=H\left(\bar{u}_{0}\right)+\frac{h}{1 !} H^{1}\left(\bar{u}_{0}\right)+\frac{h^{2}}{2 !} H^{11}\left(\bar{u}_{0}\right)+\cdots
$$

with $\bar{u}_{0}=C_{1} \cos \tau, h=C_{2} \cos 3 \tau+C_{3} \cos 5 \tau$, we can write successively

$$
\begin{aligned}
\bar{u}^{4 / 3}= & \left(C_{1} \cos \tau\right)^{4 / 3}+\frac{4}{3}\left(C_{2} \cos 3 \tau+C_{3} \cos 5 \tau\right) \\
& \times\left(C_{1} \cos \tau\right)^{1 / 3}+\cdots \\
= & \alpha C_{1}^{4 / 3} \cos \tau \\
& \times\left(\cos \tau-\frac{1}{5} \cos 3 \tau+\frac{1}{10} \cos 5 \tau-\frac{7}{110} \cos 7 \tau+\cdots\right) \\
& +\frac{4 \alpha C_{1}^{1 / 3}}{3}\left(C_{2} \cos 3 \tau+C_{3} \cos 5 \tau\right) \\
& \times\left(\cos \tau-\frac{1}{5} \cos 3 \tau+\frac{1}{10} \cos 5 \tau-\frac{7}{110} \cos 7 \tau+\cdots\right) \\
= & \alpha C_{1}^{1 / 3}\left[\frac{1}{2} C_{1}-\frac{2}{15} C_{2}+\frac{1}{15} C_{3}\right. \\
& +\left(\frac{2}{5} C_{1}-\frac{2}{3} C_{2}-\frac{3}{5} C_{3}\right) \cos 2 \tau \\
& +\left(-\frac{1}{20} C_{1}+\frac{103}{165} C_{2}+\frac{126}{165} C_{3}\right) \cos 4 \tau \\
& +\cdots],
\end{aligned}
$$

$$
\int_{0}^{\pi / 2} \bar{u}^{4 / 3} d \tau=\frac{\pi \alpha C_{1}^{1 / 3}}{2}\left(\frac{1}{2} C_{1}-\frac{2}{15} C_{2}+\frac{1}{15} C_{3}\right) .
$$

Substituting (19) and (24) into (13) we obtain

$$
\begin{aligned}
\frac{2 J}{\pi}=- & \frac{1}{4} \Omega^{2}\left(C_{1}^{2}+9 C_{2}^{2}+25 C_{3}^{2}\right) \\
+ & \frac{a \alpha C_{1}^{1 / 3}}{40 A^{2 / 3}}\left(15 C_{1}-4 C_{2}+2 C_{3}\right) \\
+ & \frac{b A^{2}}{32}\left[3\left(C_{1}^{4}+C_{2}^{4}+C_{3}^{4}\right)\right. \\
& +12\left(C_{1}^{2} C_{2}^{2}+C_{1}^{2} C_{3}^{2}+C_{2}^{2} C_{3}^{2}\right) \\
& \left.+4 C_{1}^{3} C_{2}+12 C_{1}^{2} C_{2} C_{3}+12 C_{1} C_{2}^{2} C_{3}\right] .
\end{aligned}
$$

From (25), (14), and (15) we can obtain the unknowns $C_{1}$, $C_{2}, C_{3}$, and $\Omega$.

We remark that with the approximation given by (19), there are four things to be calculated: the optimal values of the convergence-control parameters $C_{1}, C_{2}, C_{3}$, and the frequency $\Omega$. As it can be seen, the complexity of the equations is such that only numerical solutions can be found for particular values of the parameters $a, b$, and $A$, which will be further developed. 


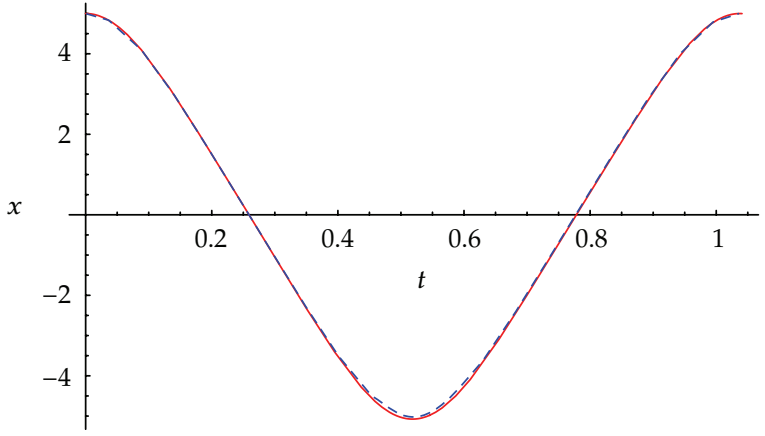

FIgURE 3: Comparison between the approximate solution (31) and numerical solution of (3) in the case $a=b=2, A=5$, red solid line: numerical integration results, blue dashed line: approximate solution.

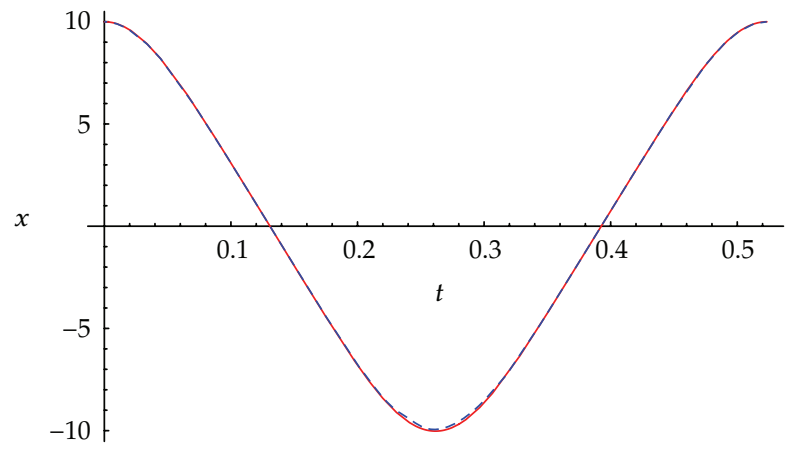

FIGURE 4: Comparison between the approximate solution (33) and numerical solution of (3) in the case $a=b=2, A=10$, red solid line: numerical integration results, blue dashed line: approximate solution.

\section{Numerical Examples}

In order to show the validity and accuracy of the OVM, we consider the following cases.

(1) In the first case we consider $a=b=1$ and $A=5$ and we obtain

$$
\begin{aligned}
& C_{1}=0.956942, \quad C_{2}=0.041274, \\
& C_{3}=0.0017835, \quad \Omega=4.287825 \text {. }
\end{aligned}
$$

The approximate solution of (3) becomes

$$
\begin{gathered}
\bar{x}(t)=4.78471 \cos \Omega t+0.20637 \cos 3 \Omega t \\
+0.0089175 \cos 5 \Omega t .
\end{gathered}
$$

The value of $\Omega$ obtained by numerical integration is $\Omega_{N}=4.281323$.

(2) In the second case, for $a=b=1$ and $A=10$ we have

$$
\begin{aligned}
& C_{1}=0.955367, \quad C_{2}=0.0427864, \\
& C_{3}=0.00184695, \quad \Omega=8.489069 \text {, }
\end{aligned}
$$

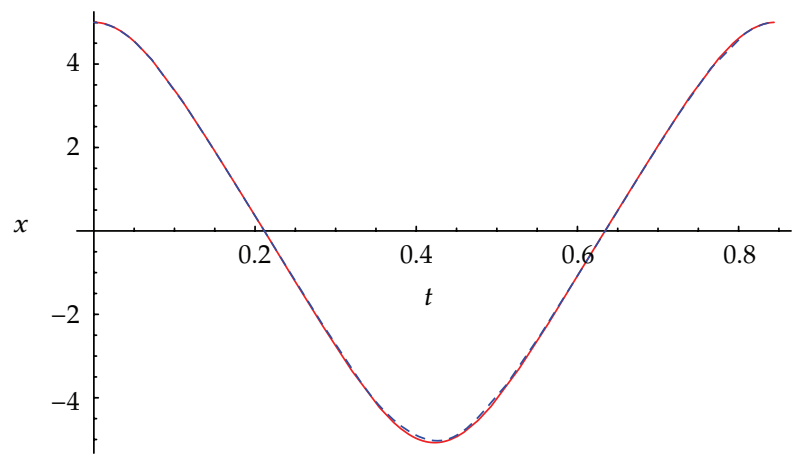

FIGURE 5: Comparison between the approximate solution (35) and numerical solution of (3) in the case $a=b=3, A=5$, red solid line: numerical integration results, blue dashed line: approximate solution.

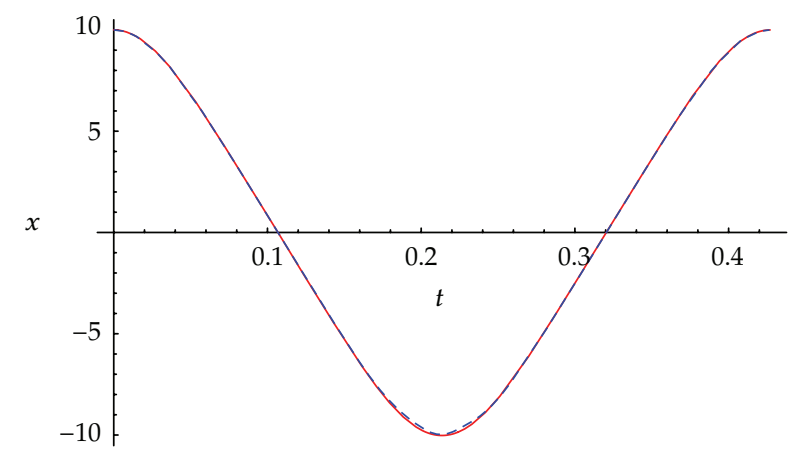

Figure 6: Comparison between the approximate solution and numerical solution of (37) in the case $a=b=3, A=10$, red solid line: numerical integration results, blue dashed line: approximate solution.

and therefore the approximate solution becomes

$$
\begin{aligned}
\bar{x}(t)= & 9.55367 \cos \Omega t \\
& +0.427864 \cos 3 \Omega t+0.0184695 \cos 5 \Omega t .
\end{aligned}
$$

In this case, the value of $\Omega$ obtained by numerical integration is $\Omega_{N}=8.485057$.

(3) In the third case, we consider $a=b=2$ and $A=5$ and thus

$$
\begin{aligned}
C_{1} & =0.956797, \quad C_{2}=0.0414033, \\
C_{3} & =0.00179965, \quad \Omega=6.063427 .
\end{aligned}
$$

The approximate solution of (3) can be written as

$$
\bar{x}(t)=4.783985 \cos \Omega t
$$

$$
+0.2070165 \cos 3 \Omega t+0.00899825 \cos 5 \Omega t \text {. }
$$

The result of numerical integration for the frequency $\Omega$ is in this case $\Omega_{N}=6.059008$. 
(4) In the case $a=b=2$ and $A=10$ we get

$$
\begin{aligned}
& C_{1}=0.955313, \quad C_{2}=0.0428326, \\
& C_{3}=0.00185462, \quad \Omega=12.00502,
\end{aligned}
$$

and the approximate solution of (3) will be

$$
\bar{x}(t)=9.55313 \cos \Omega t
$$

$$
+0.428326 \cos 3 \Omega t+0.0185462 \cos 5 \Omega t \text {. }
$$

By numerical integration, we get in this case the frequency $\Omega_{N}=12.003983$.

(5) For $a=b=3$ and $A=5$, we obtain

$$
\begin{aligned}
& C_{1}=0.956908, \quad C_{2}=0.0413049, \\
& C_{3}=0.00178722, \quad \Omega=7.426642,
\end{aligned}
$$

and the approximate solution of (3) becomes

$$
\begin{aligned}
\bar{x}(t)= & 4.78454 \cos \Omega t \\
& +0.2065245 \cos 3 \Omega t+0.00893611 \cos 5 \Omega t .
\end{aligned}
$$

In this case the numerical integration result for the frequency is $\Omega_{N}=7.434185$.

(6) In the last case, we consider $a=b=3$ and $A=10$ and therefore

$$
\begin{array}{ll}
C_{1}=0.955363, & C_{2}=0.0427894, \\
C_{3}=0.0018479, & \Omega=14.703456,
\end{array}
$$

and the approximate solution of (3) becomes

$$
\begin{aligned}
\bar{x}(t)= & 9.55363 \cos \Omega t \\
& +0.427894 \cos 3 \Omega t+0.018479 \cos 5 \Omega t .
\end{aligned}
$$

For comparison purposes, the frequency of the system obtained directly by numerical integration is $\Omega_{N}=$ 14.705077.

Figures 1, 2, 3, 4, 5, and 6 present a comparison between the present solutions (27)-(37), respectively, and the numerical integration results for (3) and (4). Thus, it is easier to emphasize the accuracy of the obtained results, since within these graphical representations, the analytical results obtained through OVM are nearly identical with numerical ones. Also, the approximate frequencies obtained by OVM are in very good agreement with those obtained by numerical integration, which also proves the validity of the approximate results.

\section{Conclusions}

In this paper we introduce the Optimal Variational Method to propose a new analytic approximate periodic solution to TNO. Our procedure is valid even if the nonlinear equation does not contain small or large parameters. OVM provides us with a simple and rigorous way to control and adjust the convergence of a solution through several convergencecontrol parameters $C_{i}$ whose values are optimally determined. This new method is very rapid and effective, and we prove it by comparing the approximate periodic solutions and frequencies obtained through the proposed method with numerical integration results. An excellent agreement has been demonstrated between the analytical and numerical integration results, and also for large values of the initial amplitudes, which validates the effectiveness of the proposed method. The proposed procedure can also be used to find analytical approximate solutions to other classes of conservative oscillators.

The main advantage of the method consists in that it provides us with a great freedom in choosing the approximate periodic solution dependent on an arbitrary number of initially unknown parameters. It is interesting to remark that unlike other known approximate methods applicable for such problems (such as the harmonic balance method, the multiple scales method, and so on), the frequency $\Omega$ of the system is not obtained imposing some conditions to avoid secular terms, which is a usual procedure within other methods, but this frequency results directly from the conditions to minimize the residual functional along with the initial conditions.

\section{Acknowledgment}

This work was supported by CNCS-UEFISCDI, Project no. PN-II-ID-PCE-2012-4-0358.

\section{References}

[1] A. H. Nayfeh and D. T. Mook, Nonlinear Oscillations, John Wiley and Sons, New York, NY, USA, 1979.

[2] P. Hagedorn, Nonlinear Oscillations, vol. 10, Clarendon Press, Oxford, UK, 1988.

[3] R. E. Mickens, Oscillations in Planar Dynamical Systems, vol. 37, World Scientific, Singapore, 1996.

[4] J.-H. He, "Modified Lindstedt-Poincare methods for some strongly nonlinear oscillators. Part.I. expansion of a constant," International Journal of Non-Linear Mechanics, vol. 37, no. 2, pp. 309-314, 2002.

[5] A. Beléndez, C. Pascual, C. Neipp, T. Beléndez, and A. Hernández, "An equivalent linearization method for conservative nonlinear oscillators," International Journal of Nonlinear Sciences and Numerical Simulation, vol. 9, no. 1, pp. 9-17, 2008.

[6] J. I. Ramos, "Linearized Galerkin and artificial parameter techniques for the determination of periodic solutions of nonlinear oscillators," Applied Mathematics and Computation, vol. 196, no. 2, pp. 483-493, 2008.

[7] G. Adomian, "A review of the decomposition method in applied mathematics," Journal of Mathematical Analysis and Applications, vol. 135, no. 2, pp. 501-544, 1988. 
[8] V. Marinca and N. Herişanu, "Determination of periodic solutions for the motion of a particle on a rotating parabola by means of the optimal homotopy asymptotic method," Journal of Sound and Vibration, vol. 329, no. 9, pp. 1450-1459, 2010.

[9] V. Marinca and N. Herişanu, Nonlinear Dynamical Systems in Engineering. Some Approximate Approaches, Springer, Berlin, Germany, 2011.

[10] N. Herişanu and V. Marinca, "A modified variational iteration method for strongly nonlinear problems," Nonlinear Science Letters A, vol. 1, pp. 183-192, 2010.

[11] J. H. He, "Preliminary report on the energy balance for nonlinear oscillations," Mechanics Research Communications, vol. 29, pp. 107-111, 2003.

[12] V. Obădeanu and V. Marinca, The Inverse Problem in Analytic Mechanics, University of Timişoara, Timişoara, Romania, 1992.

[13] R. E. Mickens and K. Oyedeji, "Construction of approximate analytical solutions to a new class of nonlinear oscillator equation," Journal of Sound and Vibration, vol. 162, no. 4, pp. 579-582, 1985.

[14] R. E. Mickens, "A generalized iteration procedure for calculating approximations to periodic solutions of 'truly nonlinear oscillators,' Journal of Sound and Vibration, vol. 287, no. 4-5, pp. 10451051, 2005.

[15] R. E. Mickens, "Iteration method solutions for conservative and limit-cycle $\mathrm{X}^{1 / 3}$ force oscillators," Journal of Sound and Vibration, vol. 292, no. 3-5, pp. 964-968, 2006. 


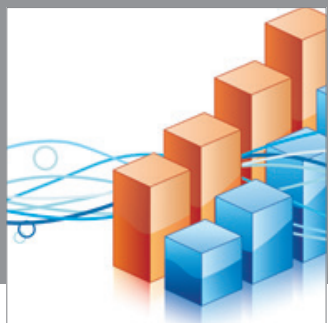

Advances in

Operations Research

mansans

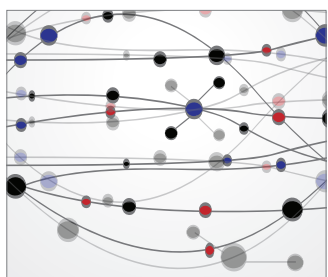

The Scientific World Journal
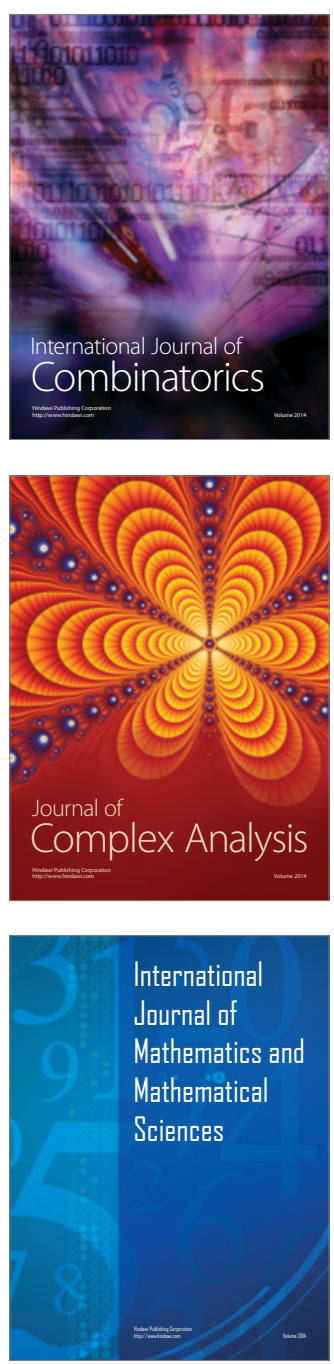
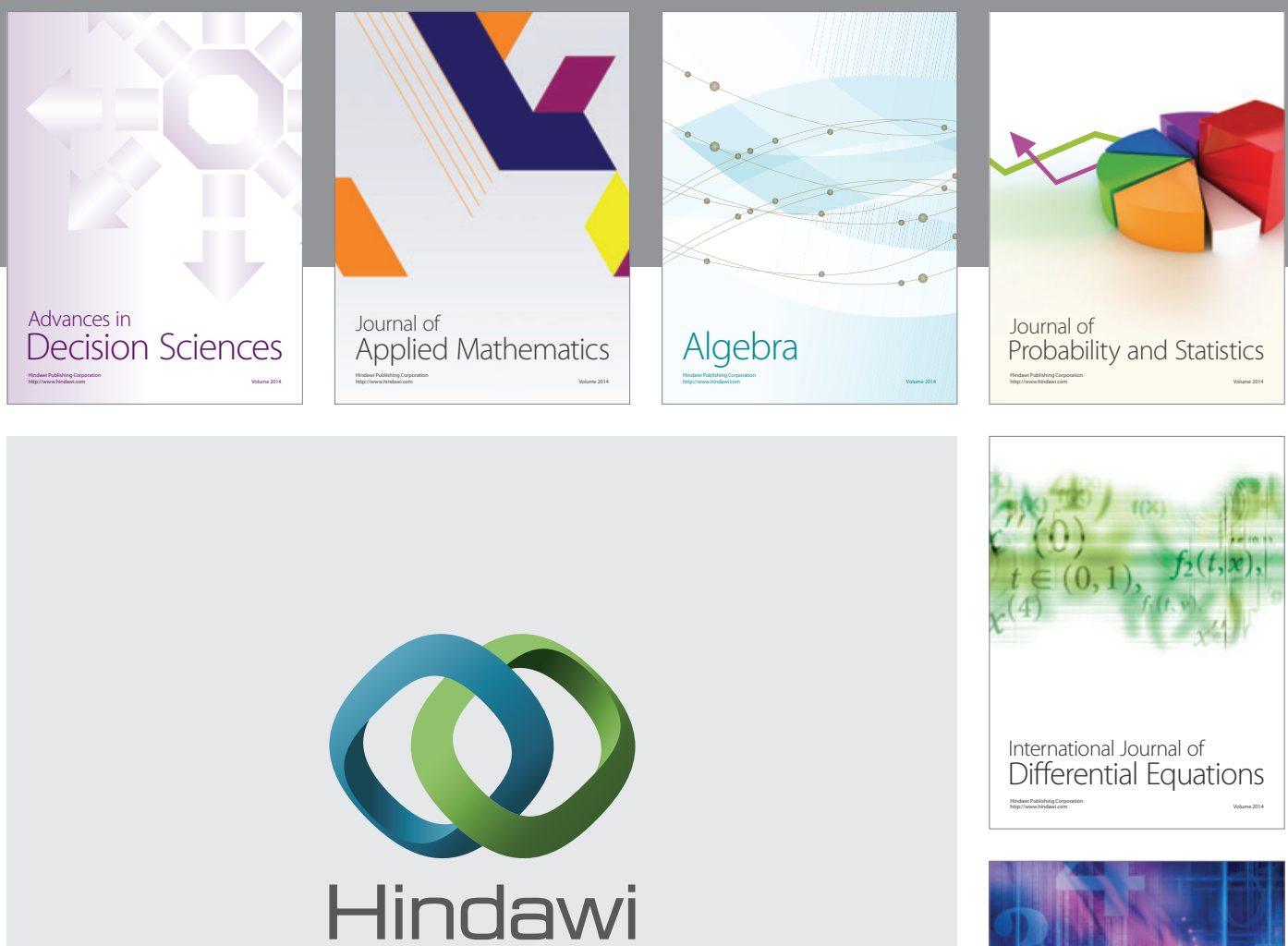

Submit your manuscripts at http://www.hindawi.com
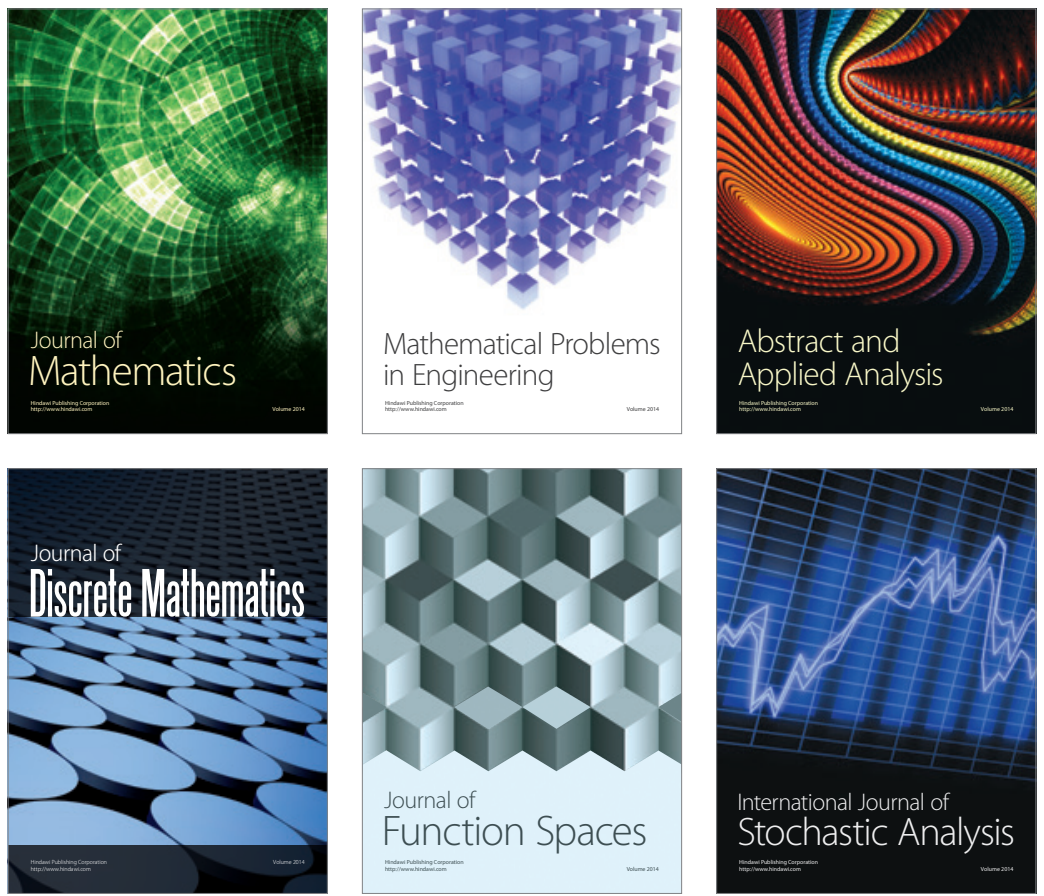

Journal of

Function Spaces

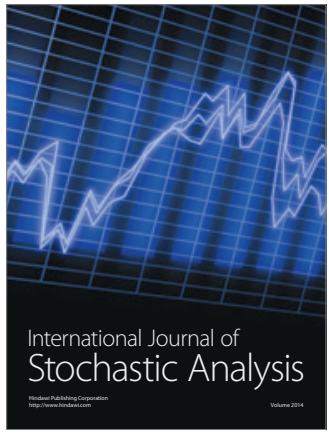

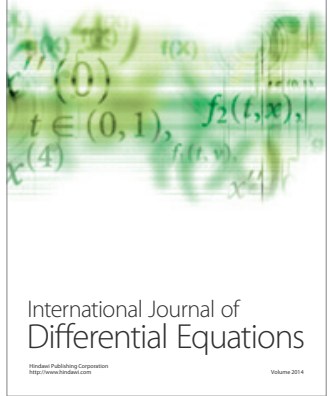
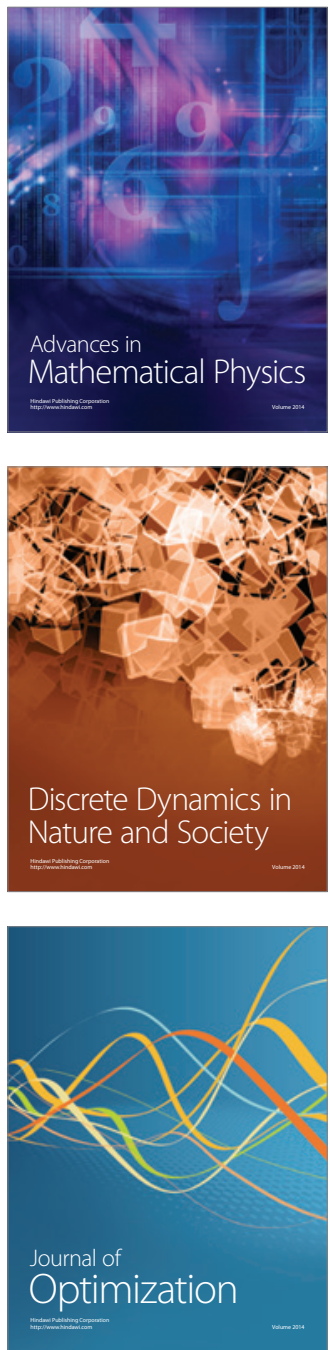